\title{
Analytical Studies of Probe Conduction Errors in Ground Temperature Measurements
}

\author{
B. A. Peavy \\ Institute for Applied Technology, National Bureau of Standards, Washington, D.C. 20234
}

(August 27, 1968)

\begin{abstract}
Vertical probes with temperature sensing elements placed at fixed positions along their length are commonly used for measuring earth temperature variations with time. Mainly for structural reasons, the probes are comprised of materials whose thermal properties are not the same as those of the surrounding earth, so that the temperatures as measured at a given time by the probe are not the same as that for the undisturbed earth. A mathematical analysis for steady periodic, two-dimensional heat flow in a two body composite has been made to determine the probe conduction errors in ground temperature measurements. Several examples are given to show the relative magnitude of probe conduction errors.
\end{abstract}

Key Words: Earth temperature; probe conduction errors; steady periodic heat flow.

\section{Introduction}

The diurnal and annual variation of the temperatures in earth or soil at varying depths beneath the ground surface has a considerable significance in a wide variety of applications. These include the determination of thermal properties of undisturbed soils from temperature measurements, the depth at which water and sewer pipes have to be buried to prevent damage by freezing, the effect of temperature variations on heat losses from buried utility pipes and the design of house foundations, and the need to know the temperature as a function of time and depth adjacent to unoccupied underground cavities in order to evaluate heat transfer in the earth from time of occupancy.

The accurate measurement of temperature variations demands that some attention be given to the method of measurement. Some of the considerations necessary for the method are that the earth be relatively undisturbed by the insertion of temperature sensing elements (usually thermocouples, resistance thermometers or thermistors), and that the depth of the element below the surface of the earth be known quite accurately. These considerations are usually met by attaching thermocouples securely at fixed positions along the length of a rigid probe (pipe or tube) before inserting the probe into the earth. The positions or depths below the surface of the thermocouples are then known quite accurately. Other considerations for the accurate measurement of temperature are the protection of the temperature sensing elements from corrosion and stray electric fields.
The probe which is inserted into the undisturbed earth must be sufficiently strong and rigid that it not be deformed or broken upon insertion. This structural requirement may not be compatible with the requirement that the thermal properties of the probe material be similar to the thermal properties of the surrounding earth. Under the influence of temperature variations at the surface of the earth, appreciable differences in thermal properties will lead to errors in the measured variation of temperature.

The purpose of this paper is to show the effect on temperature variations at depths below the surface by placing a vertical probe in the earth.

\section{Mathematical Analysis}

This analysis of steady, periodic, two-dimensional heat flow assumes a simple model such as shown in figure 1 , where a sine-wave temperature variation is applied to the surface, $z=0$, and at a sufficient depth, $z=l$, below the surface, the temperature is invariant with time. Region 1 comprises the earth with a thermal conductivity $\lambda_{1}$ and thermal diffusivity $\alpha_{1}$. Region 2 is comprised of the probe material with a thermal conductivity $\lambda_{2}$ and thermal diffusivity $\alpha_{2}$, and is in perfect thermal contact with region 1 . Other more complex models and boundary conditions can be as. sumed using a similar analysis.

For a cylindrical geometry with the axis of the system at the center of region 2 (fig. 1), the partial differential equation for heat conduction in two regions is 


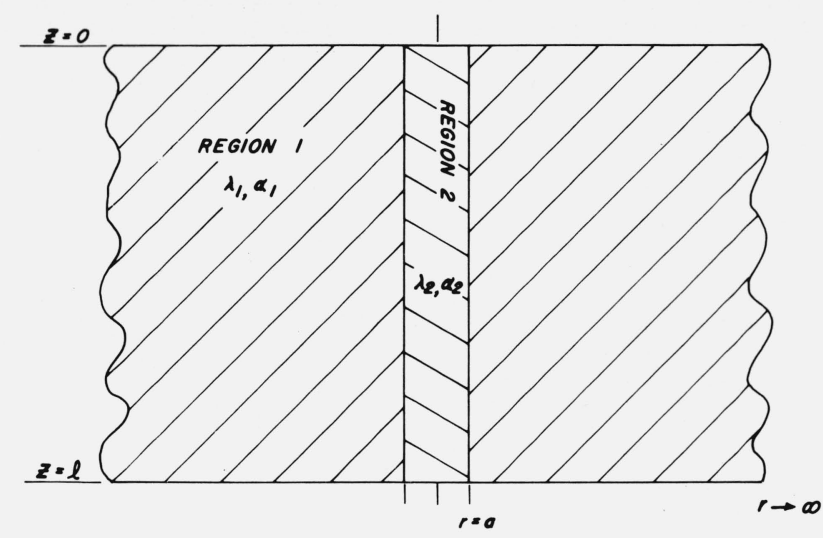

FigURE 1. Model for steady periodic heat flow in earth (region 1) shown with probe (region 2) of radius, $r=a$.

At surface $z=0$ temperature varies sinusoidally and at $z=l$ the temperature is invariant with time.

$$
\frac{\partial^{2} v_{i}}{\partial r^{2}}+\frac{1}{r} \frac{\partial v_{i}}{\partial r}+\frac{\partial^{2} v_{i}}{\partial z^{2}}=\frac{1}{\alpha_{i}} \frac{\partial v_{i}}{\partial t}
$$

where $v$ is the temperature potential measured from the temperature at $z=l, \alpha$ is the thermal diffusivity and subscript $i$ denotes either region 1 or 2 . There are several methods by which this problem may be solved and the author has found it convenient to use the Laplace transform method. The Laplace transform of (1) is

$$
\frac{\partial^{2} \bar{v}_{i}}{\partial r^{2}}+\frac{1}{r} \frac{\partial \bar{v}_{i}}{\partial r}+\frac{\partial^{2} \bar{v}_{i}}{\partial z^{2}}=q_{i}^{2} \bar{v}_{i}
$$

where $q_{i}^{2}=p / \alpha_{i}$ and $p$ is the parameter of the Laplace transform.

For region 1 , the radial temperature gradient is assumed to approach zero for very large values of the radius, $r$. The boundary conditions and continuity conditions at the interface of the two regions (fig. 1) with their respective Laplace transforms are as follows:

Surface

Condition

Transform

$$
\begin{array}{llll}
z=0 & 0 \leqslant r<\infty & v_{1}=v_{2}=\sin \omega t & \bar{v}_{1}=\bar{v}_{2}=\frac{\omega}{p^{2}+\omega^{2}} \\
z=l & 0 \leqslant r<\infty & v_{1}=v_{2}=0 & \bar{v}_{1}=\bar{v}_{2} \\
r=a & 0 \leqslant z \leqslant l & v_{1}=v_{2} & \bar{v}_{1}=\bar{v}_{2} \\
r=a & 0 \leqslant z \leqslant l & \lambda_{1} \frac{d v_{1}}{d r}=\lambda_{2} \frac{d v_{2}}{d r} & \lambda_{1} \frac{d \bar{v}_{1}}{d r}=\lambda_{2} \frac{d \bar{v}_{2}}{d r}
\end{array}
$$

where $\omega=2 \pi / T$, and $T$ is the period of a cycle. Solutions of (2) that satisfy (3) and (4) for the two regions are

$\bar{v}_{1}=\frac{\omega}{p^{2}+\omega^{2}} \frac{\sinh q_{1}(l-z)}{\sinh q_{1} l}$

$$
+\sum_{n=1}^{\infty} \frac{A_{n} K_{0}\left(\gamma_{n} r\right)}{K_{0}\left(\gamma_{n} a\right)} \sin \frac{n \pi z}{l}
$$

$\bar{v}_{2}=\frac{\omega}{p^{2}+\omega^{2}} \frac{\sinh q_{2}(l-z)}{\sinh q_{2} l}+\sum_{n=1}^{\infty} \frac{B_{n} I_{0}\left(\beta_{n} r\right)}{I_{0}\left(\beta_{n} a\right)} \sin \frac{n \pi z}{l}$

where $\gamma_{n}^{2}=q_{1}^{2}+(n \pi / l)^{2}, \beta_{n}^{2}=q_{2}^{2}+(n \pi / l)^{2}, I_{0}$ and $K_{0}$ are zero order modified Bessel functions of the first and second kind respectively, and $A_{n}$ and $B_{n}$ are constants to be determined from (5) and (6). Substitution of (7) and (8) at $r=a$ in (5), yields

$$
\begin{aligned}
\sum_{n=1}^{\infty}\left(A_{n}-B_{n}\right) \sin \frac{n \pi z}{l}= & \frac{\omega}{p^{2}+\omega^{2}} \\
& {\left[\frac{\sinh q_{2}(l-z)}{\sinh q_{2} l}-\frac{\sinh q_{1}(l-z)}{\sinh q_{1} l}\right] . }
\end{aligned}
$$

Multiplying both sides by $\sin \frac{k \pi z}{l}(k=1,2 \ldots)$ and integrating from $z=0$ to $l$, gives

$$
\begin{aligned}
A_{n}-B_{n}=\frac{2 \omega n \pi\left(q_{1}^{2}-q_{2}^{2}\right)}{l^{2}\left(p^{2}+\omega^{2}\right) \gamma_{n}^{2} \beta_{n}^{2}} & \\
= & \frac{2 \omega l^{2} p\left[\frac{1}{\alpha_{1}}-\frac{1}{\alpha_{2}}\right]}{n^{3} \pi^{3}\left(p^{2}+\omega^{2}\right)(1+p d)(1+p c)}
\end{aligned}
$$

where $d=l^{2} / \alpha_{2} n^{2} \pi^{2}$ and $c=l^{2} / \alpha_{1} n^{2} \pi^{2}$. Differentiation of (7) and (8) with respect to $r$ and substitution in (6) yields

$$
B_{n}=-\frac{A_{n} \lambda_{1} \gamma_{n} K_{1}\left(\gamma_{n} a\right) I_{0}\left(\beta_{n} a\right)}{\lambda_{2} \beta_{n} K_{0}\left(\gamma_{n} a\right) I_{1}\left(\beta_{n} a\right)} .
$$

Substitution of (10) in (9) gives $A_{n}$, which when substituted in (7) gives at the interface, $r=a$,

$$
\begin{aligned}
\bar{v}_{1}(a)=\frac{\omega}{p^{2}+\omega^{2}} & \frac{\sinh q_{1}(l-z)}{\sinh q_{1} l} \\
& +\frac{2 \omega l^{2} p\left[\frac{1}{\alpha_{1}}-\frac{1}{\alpha_{2}}\right]}{p^{2}+\omega^{2}} \sum_{n=1}^{\infty} \frac{\sin n_{\pi} z / l}{n^{3} \pi^{3} W(p)}
\end{aligned}
$$

where

$$
\begin{aligned}
W(p) & =(1+p c)(1+p d)\left\{1+\frac{\lambda_{1}(1+p c)^{1 / 2} I_{0}(L) K_{1}(J)}{\lambda_{2}(1+p d)^{1 / 2} I_{1}(L) K_{0}(J)}\right\} \\
L & =\frac{n \pi a}{l}(1+p d)^{1 / 2} \\
J & =\frac{n \pi a}{l}(1+p c)^{1 / 2} .
\end{aligned}
$$

To obtain $\mathrm{v}_{1}$ from $\bar{v}_{1}$, the Inversion Theorem for the Laplace transformation is used, where for determining the steady periodic portion only the simple poles at $p= \pm i \omega$ need be considered. The residues at the poles yield

$v_{1}(a)=[(X R+Y S) \sin \omega t+(X S-R Y) \cos \omega t] /\left(X^{2} Y^{2}\right)$ 
$+2 \omega l^{2}\left[1-\frac{\alpha_{1}}{\alpha_{2}}\right] \sum_{n=1}^{\infty} \frac{(P \cos \omega t+Q \sin \omega t) \sin n \pi z / l}{n^{3} \pi^{3}\left(P^{2}+Q^{2}\right)}$

where $\psi=\sqrt{\pi /\left(\alpha_{1} T / l^{2}\right)}$

$$
\begin{aligned}
& X=\sinh \psi \cos \psi \\
& Y=\sin \psi \cosh \psi \\
& R=\sinh \psi(1-z / l) \cos \psi(1-z / l) \\
& S=\sin \psi(1-z / l) \cosh \psi(1-z / l) \\
& W( \pm i \omega)=P \pm i Q
\end{aligned}
$$

The real and imaginary parts of $W$ are determined using complex arithmetic where the real and imaginary parts of the Bessel functions of complex argument are given by

$$
\begin{gathered}
I_{n}\left(\rho e^{i \varphi}\right)=M_{n}(\rho, \varphi)+i N_{n}(\rho, \varphi) \\
K_{n}\left(\rho e^{i \varphi}\right)=G_{n}(\rho, \varphi)+i H_{n}(\rho, \varphi) .
\end{gathered}
$$

A computer subroutine has been programmed to give values for $M_{n}, N_{n}, G_{n}$, and $H_{n}$ for $n=0$ and 1 . Numerical values for the temperature potential at the interface between the probe and the earth have been calculated from (12) using a Univac 1108 digital computer.

\section{Numerical Solutions}

If the thermal diffusivities of the earth and the probe material are equal, the last term on the right side of (12) goes to zero and temperature variations with time and depth from the surface will be the same at corresponding depths anywhere in the earth.

For numerical solutions, there are numerous possible variations of the parameters. For this reason, only the effects of variations in the thermal properties of the probe material (region 2) will be studied while the other parameters will be held constant. For a diurnal temperature variation, let

$T=24 \mathrm{hr}(86400 \mathrm{~s})$

$$
l=5 \mathrm{ft}(1.524 \mathrm{~m})
$$

$$
\begin{aligned}
a & =0.05 \mathrm{ft}(0.01524 \mathrm{~m}) \\
\lambda_{1} & =0.21 \mathrm{BTU} \mathrm{hr}^{-1} \mathrm{ft}^{-1}(\operatorname{deg~F})^{-1}\left(0.3633 \mathrm{w} \mathrm{m}^{-1} \mathrm{~K}^{-1}\right) \\
\alpha_{1} & =0.01 \mathrm{ft}^{2} \mathrm{hr}^{-1}\left(2.58 \times 10^{-7} \mathrm{~m}^{2} \mathrm{~s}^{-1}\right) .
\end{aligned}
$$

Because the probe may not be comprised of a homogeneous material and in most cases will be a filled tube, its thermal properties are difficult to specify. For this reason, four sets of "effective" thermal properties were investigated characterizing undisturbed earth and low, medium and high thermal conductivity probe materials. The thermal properties for case 2 may approximate those for wood or bakelite, for case 3, stone or masonry and for case 4, stainless steel.

Numerical solutions of $v_{1}$ against time (12) for the four cases are shown in figure 2. Curves are shown for the 2 in $(0.0508 \mathrm{~m}), 6$ in $(0.1524 \mathrm{~m})$ and 12 in $(0.3048 \mathrm{~m})$ depths and for only a $12 \mathrm{hr}$ portion of the $24 \mathrm{hr}$ cycle. Since the amplitude of the temperature potential was assumed to be unity (3), the actual temperature $\theta(a, z, t)$ at the radius $r=a$ as a function of time and position may be found from the ratio

$$
v_{1}(a, z, t)=\frac{\theta(a, z, t)-\theta_{1}}{\theta_{2}}
$$

where $\theta_{1}$ is the temperature at $z=l$ and $\theta_{2}$ is the amplitude of the temperature variation at $z=0$.

In case 1 , the probe material has the same properties as the surrounding earth and therefore the temperature variation is the same as that for undisturbed earth under the boundary conditions cited. Temperature variations for cases 2 and 3 give negligibly small deviation from the undisturbed earth temperature at the 12 in depth. Case 4 representing a highly conductive probe shows large deviations from the undisturbed earth temperature at these depths. With this probe there is a considerable phase angle deviation which increases with increase in depth below the surface.

Figures $3 \mathrm{a}, 3 \mathrm{~b}$, and $3 \mathrm{c}$ show the temperature differences between the temperature potential at $r=a$ and that for the undisturbed earth for case 2,3 , and 4 as a function of time and position below the surface $z=0$. Letting $\Delta$ be the temperature difference as described above, then the actual temperature difference between the temperature of the probe at $r=a$ and that for the undisturbed earth is

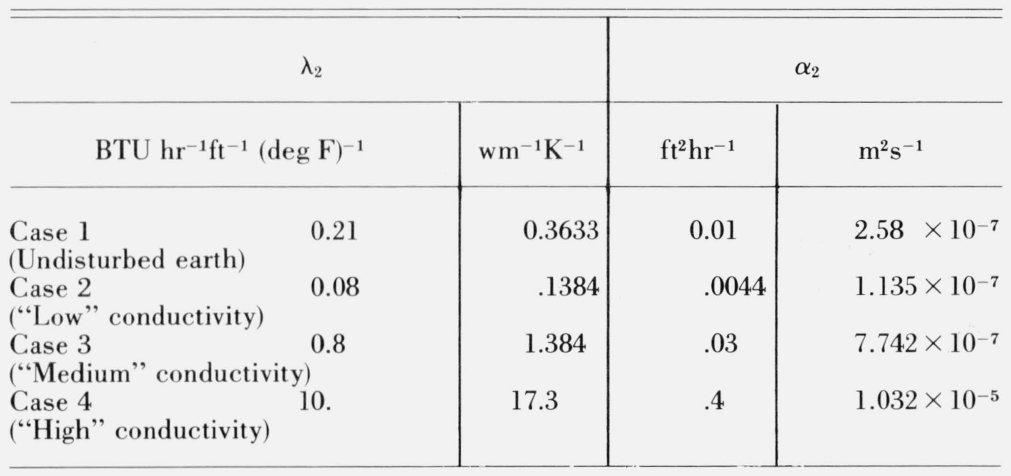




$$
\Delta_{a}=\theta_{2} \Delta .
$$

Assuming a $10{ }^{\circ} \mathrm{F}$ amplitude for the diurnal variation, then for case 4 at $t=6 \mathrm{hr}$ the actual temperature difference is $2.26{ }^{\circ} \mathrm{F}$ for the 2 in depth, $3.06{ }^{\circ} \mathrm{F}$ for the 4 in depth, $2.66{ }^{\circ} \mathrm{F}$ for the 6 in depth and $0.45^{\circ} \mathrm{F}$ for 12 in depth.

\section{Discussion}

The mathematical development given in a previous section considers a pure sine-wave temperature variation at the surface of the earth, whereas in nature

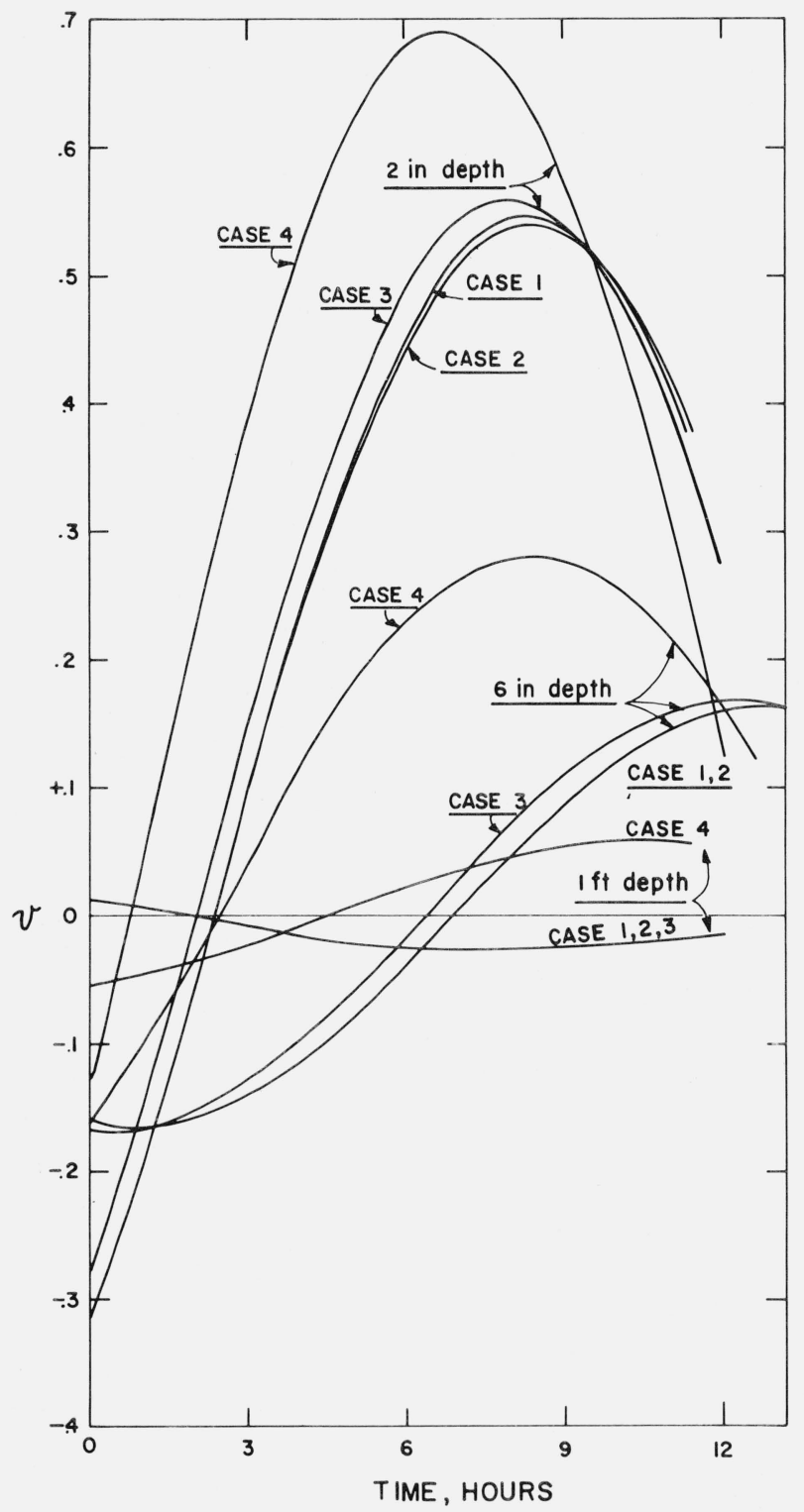

FigurE 2. Temperature variation with time and depth below the surface at the radius $\mathrm{r}=\mathrm{a}$ ( $f$ g. 1 ) for the four cases, assuming unit amplitude in the temperature variation at the depth $\mathrm{z}=0$. the actual surface temperature variation for a weather cycle is given by the trigonometric series

$$
v=\sum_{m=1}^{\kappa} B_{m} \sin \left(\omega_{m} t-\varphi_{m}\right)
$$
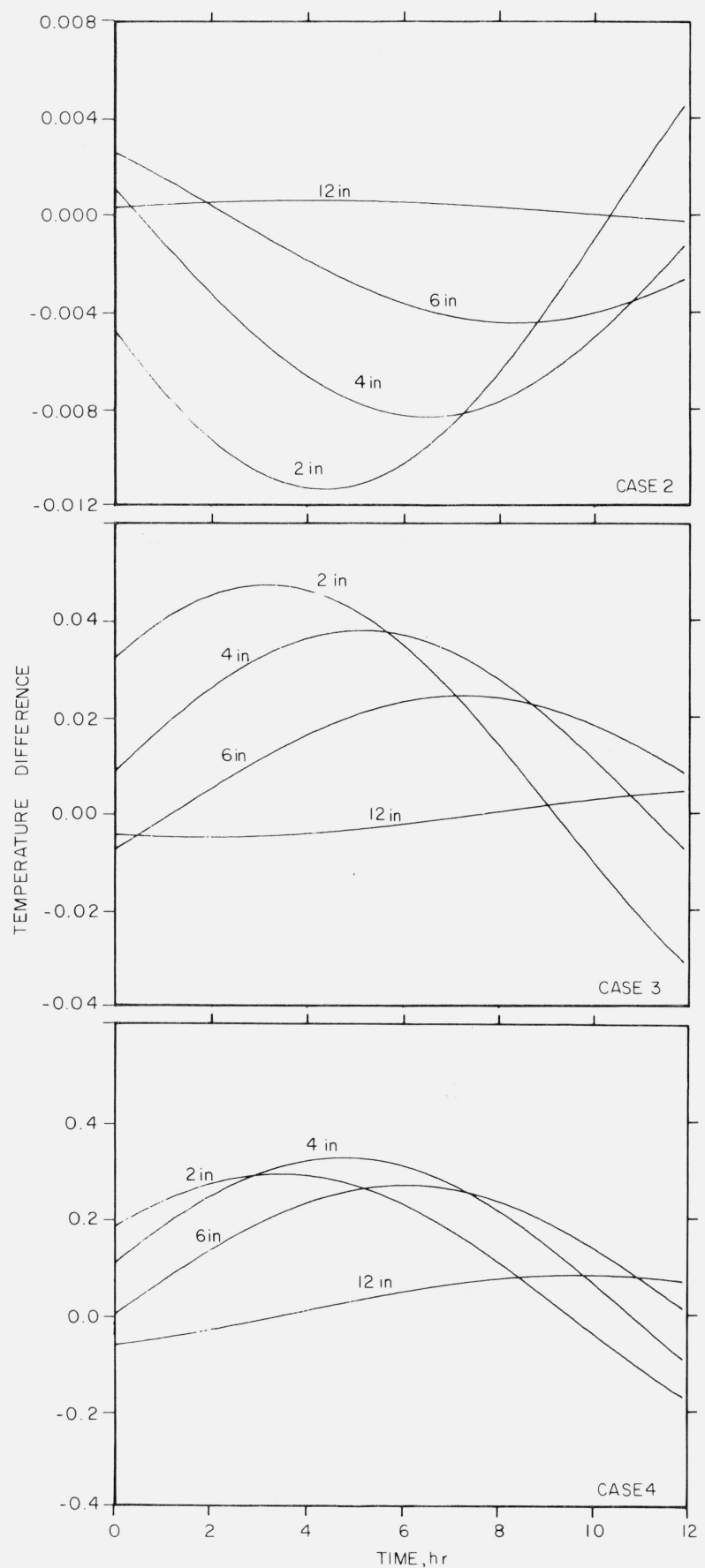

FIGURE 3. Temperature difference between temperature at $\mathrm{r}=\mathrm{a}$ and that for undisturbed earth for (a) case 2, (b) case 3 and (c) case 4 as a function of time and position below the surface $\mathrm{z}=0$. 
where $\kappa$ can be considered to be a finite number. This relationship can also be satisfied in the mathematical development by its substitution for (3). This was not considered necessary for the purpose of this paper, which is to present an analytical method and to show by numerical results the relative magnitude of probe conduction errors on a diurnal basis. If an annual cycle is to be considered, the depth $l$, at which the temperature is assumed to be invariant with time should be much greater than the $5 \mathrm{ft}$ used for the diurnal cycle.

A study of the transient case was made by Brown. ${ }^{1}$ He performed tests which produced a monotonically increasing temperature change with time at the various depths below the surface. For the usual periodic

${ }^{1}$ W. G. Brown, Thermal model tests for probe conduction errors in ground temperature measurement (Res. Paper 194, Division of Building Research, National Research Council. Canada, Sept. 1963) weather cycle (diurnal and annual), the present analysis permits estimation of the magnitude of the harmonically varying probe conduction errors represented by the differences in temperature when employing probes of different thermal properties (cases 2, 3, and 4) compared to that of undisturbed earth (case 1).

The impetus for this paper is the determination of the thermal properties of undisturbed soils or earth from ground temperature measurements. This work is currently sponsored by the Office of Civil Defense, Department of the Army, and consists of this analysis, laboratory tests of probe configurations under timevarying temperature conditions, and actual field measurements now underway.

(Paper 72C4-281) 